Nouvelles perspectives en sciences sociales

Revue internationale de systémique complexe et d'études relationnelles

\title{
La double quête : un essai sur la dimension cosmologique de synthèses interdisciplinaires en sciences sociales
}

\section{Wiktor Stoczkowski}

Volume 7, numéro 1, octobre 2011

Sur le thème de l'interdisciplinarité

URI : https://id.erudit.org/iderudit/1007085ar

DOI : https://doi.org/10.7202/1007085ar

Aller au sommaire du numéro

Éditeur(s)

Prise de parole

ISSN

1712-8307 (imprimé)

1918-7475 (numérique)

Découvrir la revue

Citer cet article

Stoczkowski, W. (2011). La double quête : un essai sur la dimension cosmologique de synthèses interdisciplinaires en sciences sociales. Nouvelles perspectives en sciences sociales, 7(1), 137-155.

https://doi.org/10.7202/1007085ar

\section{Résumé de l'article}

Depuis leur émergence, les sciences sociales se livrent à une double quête : d'une part, elles cherchent à construire une connaissance objective du monde humain; d'autre part, elles souhaitent employer cette connaissance pour apporter au monde humain des améliorations radicales. Ces deux ambitions ont été systématiquement tenues pour complémentaires et compatibles : la connaissance de l'homme et de la société devait être un moyen; la transformation de l'homme et de la société en était la fin. Il s'agit là d'un pari fondateur des sciences sociales, responsable de la plupart des espoirs dont on a pu les investir. En prenant comme exemple quatre " grandes théories ", cet article se propose d'analyser les présupposés et les implications de cette double quête des sciences sociales. 


\title{
La double quête : un essai sur la dimension cosmologique de synthèses interdisciplinaires en sciences sociales ${ }^{1}$
}

\author{
WikToR STOczkowskI \\ École des hautes études en sciences sociales, Paris
}

e concept d'interdisciplinarité, ainsi que l'idéal d'une colla-
boration entre diverses disciplines de la recherche, présupposent que les efforts des spécialistes, en dépit de tout ce qui sépare la pratique de leurs métiers respectifs, concourent à un objectif commun. Cet objectif serait une représentation unifiée et globale de la réalité. Une telle représentation est censée résulter d'une synthèse à venir des connaissances positives que chaque discipline académique échafaude séparément des autres, mais dont il est souhaitable voir un jour une convergence interdisciplinaire. Cependant, nul n'ignore que les chercheurs n'ont jamais attendu que l'accumulation patiente de données empiriques fournisse une base sûre d'une synthèse interdisciplinaire, et qu'ils se sont empressés, depuis des siècles, à en proposer diverses versions. Quelles sont les fondations de ces synthèses? Quelles sont leurs finalités? C'est à ces questions que je me propose d'esquisser ici une réponse, en me penchant sur le cas des sciences sociales.

Il s'agit du texte remanié de la conférence donnée au Centre interuniversitaire de recherche sur la science et la technologie de l'Université du Québec à Montréal, le 11 juin 2010. 
L'étude des sciences constitue un domaine déjà solidement établi et extrêmement riche. Malgré leur grande variété, les recherches menées dans ce domaine restent encore fortement focalisées sur les sciences exactes et naturelles. Quant aux sciences sociales, elles gardent le statut d'instrument d'observation et d'interprétation, à l'abri du type d'analyse à laquelle elles soumettent les autres disciplines scientifiques.

Ce n'est pas que les sciences sociales soient avares de commentaires sur elles-mêmes. Bien au contraire, on assiste à un véritable flot de gloses que chaque discipline des sciences sociales produit, selon des routines consacrées, pour décrire son histoire, sa vocation, ses pratiques, ses théories, sa diversité, ses enjeux sociaux. Beaucoup de chercheurs pensent qu'il est inutile d'adjoindre une voix de plus à ce vacarme de commentaires réflexifs, tantôt parce que tout a déjà été dit, tantôt parce qu'il n'y a rien particulièrement d'intéressant à en dire : c'est précisément ici que se rencontrent les certitudes de ceux qui pensent avoir tout compris et de ceux persuadés qu'il n'y a là rien à comprendre.

Je ne partage pas ces certitudes. J'estime que la majeure partie de ces discours prétendument réflexifs ne cherche nullement à décrire, et encore moins à expliquer ce que font réellement les sciences sociales. Ces discours sont plutôt mis au service des positionnements sociaux et théoriques dont l'orientation est destinée davantage à favoriser le succès de ceux qui les tiennent, qu'à analyser rigoureusement leurs pratiques. On a parfois l'impression que les sciences sociales, paradoxalement, demeurent un terrain le moins exploré du champ des études sur les sciences. S'il en est ainsi, ce n'est pas parce qu'on a insuffisamment éclairé ce domaine, mais parce que, au contraire, on l'a surexposé : de même que sur une pellicule photographique, l'image peut être inintelligible aussi bien par un défaut que par un excès de lumière. Ce n'est donc ni par présomption ni par ignorance que je considère qu'il faut reprendre une étude des sciences sociales par le début, en commençant par des questions élémentaires, à la fois simples et fondamentales. Parmi celles-ci, la plus importante touche aux objectifs dont les sciences sociales se proposent, 
de manière constante, la réalisation lorsqu' elles aspirent à nous offrir une représentation synthétique et interdisciplinaire de la chose humaine.

Partons d'un constat historique. Les sciences sociales constituent une innovation relativement récente : elles n'ont été institutionnalisées qu'à partir du milieu du XIX $\mathrm{X}^{\mathrm{e}}$ siècle. Dès leurs débuts, ces sciences se sont engagées dans une double quête. Les projets qui leur ont permis de gagner rapidement une légitimité comportaient une double visée : d'une part, construire une connaissance objective du monde humain; d'autre part, employer cette connaissance pour apporter au monde humain des améliorations radicales.

Ces deux ambitions ont été systématiquement tenues pour complémentaires et compatibles : la connaissance de l'homme et de la société devait être un moyen; la transformation de l'homme et de la société en était la fin. Il s'agit là d'un pari fondateur des sciences sociales, responsable de la plupart des espoirs dont on a pu les investir. Pourtant, les présupposés et les implications de ce pari n'ont jamais été soumis à un examen approfondi. Il est indispensable d'analyser toutes les conséquences de cette double quête des sciences sociales qui aspirent simultanément à bâtir une connaissance empirique de la réalité humaine et à transformer profondément cette réalité.

Bien évidemment, il n'est pas question de balayer superficiellement cet immense domaine à travers toutes les époques et tous les pays. Après avoir effectué des études exploratoires dans plusieurs contextes disciplinaires (tels que l'anthropologie culturelle, l'histoire, la sociologie, l'archéologie), dans plusieurs contextes nationaux (France, Grande-Bretagne, États-Unis), et à plusieurs époques (seconde moitié du $\mathrm{XIX}^{\mathrm{e}}$ siècle, première et seconde moitié du XX $\mathrm{XX}^{\mathrm{e}}$ siècle), j'ai choisi de limiter mon échantillon, pour le besoin de cet essai, à un seul de ces multiples contextes, à savoir aux sciences sociales en France, au XX $\mathrm{X}^{\mathrm{e}}$ siècle. Afin de circonscrire encore davantage ma base de données, j’ai décidé de ne retenir qu'une catégorie particulière des constructions conceptuelles proposées par les sciences sociales : ce que l'on appelle les 
" grandes théories ", constructions interdisciplinaires par excellence. Ces grandes théories m'intéressent particulièrement non parce qu'elles seraient représentatives de tous les produits auxquels aboutit le travail des sciences sociales, mais parce qu'elles constituent indubitablement les produits les plus prestigieux de ces disciplines : ce sont ces grandes théories qui sont largement citées, célébrées, admirées, parfois contestées; ce sont elles qui alimentent les débats et les polémiques; ce sont elles qui suscitent de nouvelles vocations, servent de modèles, et valent aux sciences sociales tantôt des éloges dithyrambiques, tantôt de violentes réprobations. Il y a quelque chose d'important qui se joue autour de la production et de la réception de ces théories.

Je travaille donc sur un échantillon, chronologiquement et géographiquement circonscrit. Les conclusions que j'en tire valent d'abord pour ce contexte particulier. J'espère néanmoins que ces conclusions pourront trouver par la suite des applications au-delà du domaine initial où elles sont formulées : cette transposition les soumettra à une épreuve empirique, nécessaire pour évaluer leur véritable portée.

L'échantillon dont je vais traiter ici est composé de quatre "grandes théories " qui ont vu le jour en France, entre le début et la fin du XX' ${ }^{\mathrm{e}}$ siècle. Il s'agit des œuvres d'Émile Durkheim, de Claude Lévi-Strauss ${ }^{2}$, de Pierre Bourdieu et de Bruno Latour. Quatre auteurs, quatre styles, quatre méthodes, quatre moments historiques différents, et néanmoins une caractéristique commune : chacune de ces vastes synthèses théoriques veut offrir une explication interdisciplinaire et globale de la chose humaine. Bien qu'aucune d'elles ne soit parvenue à devenir un paradigme consensuel des sciences sociales, elles ont toutes gagné une ample reconnaissance, à la fois dans le milieu académique et auprès du grand public. Cette réception favorable - qui n' exclut nullement des critiques, souvent virulentes, dont elles ont toutes pu être l'objet - les rend particulièrement intéressantes pour l'anthropo-

Une étude détaillée sur l'œuvre de Lévi-Strauss a déjà été publiée par Wiktor Stoczkowski sous le titre Anthropologies rédemptrices. Le monde selon LéviStrauss Paris, Hermann, 2008)les autres sont en préparation. 
logue que je suis : les théories académiques, même quand elles sont construites en accord avec toutes les règles de la science, ne tirent pas leur autorité uniquement de leur valeur objective; il ne suffit pas qu'elles soient vraies ou fondées pour être crues; elles ont également besoin de se trouver en consonance avec les opinions et représentations qui ont cours dans la société à laquelle elles s'adressent. D'ailleurs, les faveurs que cette société leur accorde sont souvent éphémères, ce qui suggère que leur succès public, au même titre que la disgrâce qui peut s'ensuivre, dépendent tout autant de la solidité de leur démonstration que des vicissitudes historiques de la doxa du sens commun qui les juge.

Bien que cet échantillon soit limité dans le temps et dans l'espace, il est suffisant pour étayer un premier constat général. Les projets d'une amélioration radicale du monde humain nécessitent, sur le plan conceptuel, que soient remplies deux conditions. Premièrement, ces projets ont besoin d'une axiologie, c'est-à-dire d'une théorie des valeurs. Cette théorie inclut, d'un côté, des valeurs négatives, à partir desquelles on définit les maux dont il conviendrait d'affranchir le monde humain, et de l'autre, des valeurs positives, employées pour concevoir l'état vers lequel il faudrait tendre.

Deuxièmement, ces projets réformateurs visent toujours à échafauder une ontologie, c'est-à-dire une théorie de l'être, dont le rôle est de tracer une frontière entre les propriétés essentielles - donc fatalement invariables - du réel, et ses propriétés accidentelles, dont on peut espérer la modification.

Ces deux premières composantes, axiologique et ontologique, sont souvent accompagnées d'une étiologie, c'est-à-dire d'une théorie des origines. Prenant la forme d'un récit, l'étiologie a pour fonction d'expliquer comment les propriétés hypothétiquement accidentelles du monde humain (par exemple: anomie, lutte des classes, rapports de domination, surpopulation, destructions du milieu naturel, etc.) en sont progressivement venues à s'ajouter aux propriétés ontologiques du monde, pour y introduire des distorsions néfastes qu'il est souhaitable d'abroger. 
Il est intéressant d'observer que ces axiologies, ontologies et étiologies, loin de pouvoir s'appuyer exclusivement sur une assise empirique, sont souvent adoptées a priori : elles constituent donc une axiomatique des sciences sociales. Les disciplines du savoir qui visent à bâtir une représentation empiriquement fondée du monde humain, lorsqu'elles cherchent parallèlement à établir des projets de transformation radicale de ce monde, sont amenées à adopter des représentations qui ne peuvent que difficilement être ancrées dans les données factuelles.

Comment appréhender ce phénomène à la fois déconcertant, complexe et encore mal étudié? L'épistémologue ou le polémiste pourraient s'en saisir pour déplorer les défaillances des «sciences molles " qui ne parviendraient pas à se hisser au niveau des " sciences exactes ", ou pour brocarder d'inacceptables « impostures » qu'il serait urgent de démystifier. Ces approches polémiques peuvent être sans doute légitimes, souvent saines, parfois même indispensables. Mon approche est toutefois différente. L'anthropologue évite de déplorer que la culture qu'il observe ne corresponde pas à l'idéal auquel ses prédilections le poussent à accorder la préférence. Même lorsque les normes et les usages qu'il découvre heurtent sa sensibilité, il accepte de les étudier tels qu'ils sont. Cette attitude permet de quitter le terrain investi de longue date par des pamphlétaires, pour porter un regard de compréhension - mais qui n'équivaut nécessairement à l'acceptation - sur la subculture spécifique qui s'est lentement sédimentée, en Occident, dans le domaine des sciences sociales. Dès lors, il devient secondaire de savoir si ces disciplines sont toujours conformes à des modèles d'épistémologie théorique, parfois forgés par elles-mêmes en guise d'idéologies justificatrices. L'essentiel est de comprendre ce que ces sciences font réellement, quels que soient leurs credos officiels.

Quand on s'astreint à adopter cette attitude, et que l'on entre dans une analyse très détaillée des constructions conceptuelles proposées par les sciences sociales, on est frappé de constater que, non contentes d'accumuler des données, théories, interprétations, explications et autres produits associés traditionnellement à 
l'activité scientifique, les synthèses interdisciplinaires proposées par les sciences sociales déploient simultanément d'immenses efforts pour élaborer des visions du monde, quelque peu analogues à celles que les anthropologues étudient en dehors de l'Occident et auxquelles ils donnent le nom de " cosmologies". Comme toutes les autres cosmologies, celles des sciences sociales possèdent également les trois composantes métaphysiques que j'ai mentionnées (axiologique, ontologique et étiologique). Ces composantes se trouvent agencées en des systèmes plus ou moins cohérents de représentations portant sur la forme, le contenu et la dynamique de l'univers qui abrite l'homme. Ainsi, les cosmologies associées aux "grands théories " interdisciplinaires des sciences sociales tendent à opérer une conjonction entre le métaphysique et le factuel, l'un et l'autre jugés nécessaires pour rendre ces théories à la fois crédibles et pertinentes aux yeux de nos contemporains. Et puisque mon échantillon couvre tout un siècle, il est loisible de retracer - dans ce cadre chronologique une évolution historique de nos représentations cosmologiques de l'homme et de la société.

Cette évolution - même observée à l'échelle d'un seul siècle - fait apparaître non seulement des transformations, mais aussi des continuités. La plus insigne est celle qui se manifeste à travers la récurrence d'une finalité matricielle de ces constructions cosmologico-empiriques que sont les "grandes théories ». Les projets d'une transformation méliorative de la chose humaine, sousjacents aux théories des sciences sociales, comportent souvent la promesse de l'abolition d'un mal quasi-ontologique, tenu pour cause première de toutes les imperfections déplorables qui sont censées affecter la vie collective des humains. En cherchant à établir le diagnostic de ce mal primaire, et en proposant les moyens de son abrogation, les théories des sciences sociales acquièrent une authentique dimension sotériologique. Je m'empresse d'ajouter que j'emploie la notion de sotériologie dans une acception beaucoup plus large que celle que lui donne le vocabulaire théologique du christianisme, où elle est réduite au péché originel et à la rédemption des âmes par l'entremise du Messie. 
On oublie que le mot latin salus, dont dérive le substantif français salut, et le mot grec saos ou sôs, dont dérive l'adjectif sôtêr, sans être limités au destin transcendant de l'âme, englobait initialement la préservation de la santé du corps, le maintien de l'équilibre mental, la conservation des routes, des habitations ou des lois qui assuraient la bonne marche de la cité ${ }^{3}$. Le sens de ce concept pouvait donc être aussi bien spirituel que matériel, individuel ou collectif, transcendent ou immanent. C'est en recourant à cette ancienne acception du terme, retenu encore à notre époque par les dictionnaires, que l'on peut discerner une ambition sotériologique même dans les théories des sciences sociales parmi les plus matérialistes, laïques et antispiritualistes. L'une des caractéristiques centrales de ces théories est une interrogation sur la possibilité d'une mise en dehors du mal de la vie humaine, à la fois collective et individuelle, ce qui fait d'ailleurs que ces théories laïques du salut dépendent étroitement des théories du mal. Prenons quelques brefs exemples.

Selon Émile Durkheim, la France et toutes les sociétés industrielles de la fin du XIX ${ }^{\mathrm{e}}$ siècle se trouvaient dans « un état de crise et de perturbation qui ne pouvait se prolonger sans danger ": livrées au déchaînement de désirs insatiables dont l'individu est la source, selon Durkheim, elles étaient en proie à l'anomie généralisée et à la "mélancolie collective morbide ". Il fallait « qu'une puissance régulatrice joue pour les besoins moraux le même rôle que l'organisme pour les besoins physiques " ". L'étude scientifique des mécanismes de la vie sociale, en Occident et chez les peuples dits primitifs, avait chez Durkheim comme finalité d'apporter un instrument de salut à nos propres sociétés : ainsi, par exemple, la connaissance des religions australiennes, révélatrice d'un " aspect essentiel et permanent » de la nature

3 Yvan Bubloz, "Augustin et Porphyre sur le salut : pour une comparaison analogique et non apologétique du christianisme et du néoplatonisme ", dans Maya Burger et Claude Calame (dir.), Comparer les comparatismes. Perspectives sur l'histoire et les sciences des religions, Paris, Edidit, 2006, p. 113-140.

4 Émile Durkheim, Le Suicide. Etude de sociologie, Paris, Alcan, 1897, p. 418420.

5 Ibid., p. 275. 
humaine, devait nous aider à créer les conditions d'une nouvelle " effervescence créatrice " qui ferait surgir les idéaux nécessaires pour sortir les Européens de la funeste période de "médiocrité morale » et de déséquilibre qui résultait de cette crise morale ${ }^{6}$.

Claude Lévi-Strauss était, lui aussi, persuadé que le monde contemporain se trouvait dans un état de grave crise. Mais son diagnostic, et le remède qu'il préconisait, étaient différents. Pour Lévi-Strauss, les maux qui nous accablent ont pour source commune la prolifération excessive de l'espèce humaine. Les effets catastrophiques qui en résultent seraient multiples : la xénophobie, les haines, l'uniformisation culturelle qui étouffe les facultés créatives de l'homme, la dévastation du milieu naturel dont nous souillons irrémédiablement les éléments et qui prépare notre propre disparition ${ }^{7}$. Un espoir existe pourtant, et c'est la connaissance ethnologique qui le porte : l'ethnologie peut éveiller notre conscience car elle étudie des sociétés qui n’ont jamais " cultivé une image de l'homme conçu comme le seigneur et maittre de la création, libre de s'octroyer des droits exorbitants sur toutes les manifestations de la nature et de la vie. Bien au contraire : leurs systèmes philosophiques sont d'accord pour faire à l'homme une place de choix dans la nature, mais à la condition qu'elle n'empiète pas sur celle des autres espèces vivantes... " L’ethnologie lévi-straussienne ne se contente donc pas de recueillir des données scientifiques:

elle a aussi une vocation salvatrice, puisqu'elle seule peut nous faire entendre « les leçons d'une sagesse dont l'Occident pourrait s'inspirer s'il voulait éviter qu'une humanité trop imbue d'elle-même, prompte à détruire tout ce qui n'est pas elle, ne dispose plus d'aucun glacis protecteur pour se prémunir contre ses propres atteintes" ".

6 Émile Durkheim, Les Formes élémentaires de la vie religieuse, Paris, Alcan, 1912, p. 610.

7 Claude Lévi-Strauss, « Race et culture ", dans Le Regard éloigné, Paris, Plon, [1971] 1983, p. 377-222.

8 Claude Lévi-Strauss, " Discours de récipiendaire à la remise du prix Erasme ", dans Praemium Erasmianum MCMLXXIII, Amsterdam, Stichting Praemium Erasmianum, 1973, p. 27.

9 Ibid., p. 28; Voir l'analyse de cette conception dans Wiktor Stoczkowski, Anthropologies rédemptrices, op. cit. 
Quant à Pierre Bourdieu, sa sociologie dépeint un monde social impitoyable et cruel, où l'homme est un loup pour l'homme, selon ses propres termes ${ }^{10}$. Les dominants oppriment les dominés réduits à " un sentiment aigu de honte corporelle et à toutes les espèces de honte culturelle ${ }^{11}$ ". Désirant de par nature accéder à une forme reconnue d'existence sociale, les acteurs sociaux

n'ont pas d'autre choix que de lutter pour maintenir ou améliorer leur position dans le champ, contribuant ainsi à faire peser sur tous les autres les contraintes, souvent vécues comme insupportables, qui naissent de la coexistence antagoniste ${ }^{12}$.

Plus brutal que la lutte des classes, cet affrontement symbolique nous enferme dans les affres d'un monde insoutenable. Seule la vraie science du social propose un moyen de salut : " en portant au jour les mécanismes sociaux qui assurent le maintien de l'ordre établi et dont l'efficacité proprement symbolique repose sur la méconnaissance de leur logique et de leurs effets "13", l'analyse sociologique permet «...d'accéder à la liberté que la connaissance des déterminismes sociaux permet de conquérir contre les déterminismes ${ }^{14}$ ". Le dispositif salvateur que Bourdieu proposait à travers sa sociologie était double. D'une part, cette sociologie, moyennant la réflexivité et l'auto-socioanalyse, devait conduire le sociologue à une anamnèse libératrice (le terme est de Bourdieu), et favoriser chez lui une sorte de supralucidité dont la puissance lui permet, lorsqu'il dit la vérité sur soi, d'énoncer également la vérité sur les autres ${ }^{15}$. D'autre part, cette sociologie pourrait acquérir des vertus en quelque sorte démiurgiques : elle serait capable non seulement de décrire ou d'expliquer la réalité sociale, mais aussi de créer une réalité sociale en transformant sa

10 Pierre Bourdieu, Leçon sur la leçon, Paris, Minuit, 1982, p. 52.

11 Pierre Bourdieu et Yvette Delsaut, "Le couturier et sa griffe : contribution à une théorie de la magie ", Actes de la recherche en sciences sociales, vol. 1, n 1, 1975, p. 36.

12 Ibid., p. 7-36.

13 Pierre Bourdieu, "Le champ scientifique ", Actes de la recherche en sciences sociales, vol. 2, $\mathrm{n}^{\text {os }} 2-3,1976$, p. 101.

14 Pierre Bourdieu, Raisons pratiques, Paris, Seuil, 1994, p. 11.

15 Pierre Bourdieu, Ce que parler veut dire, Paris, Fayard, 1982, p. 29. 
représentation ${ }^{16}$, en opérant - dit Bourdieu - une subversion cognitive, une conversion de la vision du monde ${ }^{17}$. Ainsi, la parole du sociologue pourrait devenir créatrice et, dans certaines conditions précises, de "faire advenir ce qu'elle énonce ${ }^{18}$ ", en contribuant par là-même à transformer et à améliorer radicalement la réalité sociale.

L'« anthropologie symétrique » de Bruno Latour annonce, elle aussi, un grand chambardement salvateur. D'abord celui du passage révolutionnaire de la sociologie fascinée depuis le XIX siècle par ces entités métaphysiques que sont la société et la nature, à la nouvelle sociologie qui prend pour objet les associations hybridées, mêlant des humains et des non-humains, dont les interactions sont directement données dans l'expérience empirique. Pourtant, cette refondation sociologique fait partie d'une entreprise autrement ambitieuse. Les notions de société et de nature, vieux subterfuges des antirévolutionnaires, doivent être abandonnées pour que puisse se déployer une action politique d'envergure, destinée à transformer de fond en comble l'Occident ${ }^{19}$. Le chemin de la refondation passe par la sortie de la modernité, dont la postmodernité - phase de transition utile n'a pas réussi à supprimer les tares ontologiques. L'émergence de l'anthropologie latourienne est censée parachever cette métamorphose. Promise à aboutir à " un nouveau 'logos', une nouvelle science totale de l'homme assemblé ", elle veut aider l'Occident à repenser "sa manière de se présenter au reste du monde " et de cohabiter avec les non-humains ${ }^{20}$; elle pourra nous apprendre clame Latour - à partager un cosmos nouveau et pacifié qu'il nous faut construire, dans un effort constant de dialogue et de négociation avec une pluralité d'êtres et de cultures de la planète entière $^{21}$.

$\overline{16}$ Pierre Bourdieu, "Genèse et structure du champ religieux ", Revue française de sociologie, vol. 12, $\mathrm{n}^{\circ} 3,1971$, p. 317.

17 Pierre Bourdieu, Ce que parler veut dire, op. cit., p. 150.

18 Ibid.

19 Bruno Latour, Changer de société - refaire de la sociologie, Paris, La Découverte, 2006.

20 Ibid.

21 Bruno Latour, Un Monde pluriel mais commun, entretiens avec François Ewald, 
L'espace réduit d'un essai ne me permet de donner de ces cosmologies rédemptrices qu'un aperçu très sommaire. Je ne fais ainsi que signaler l'existence d'un phénomène qui nécessite une analyse incomparablement plus détaillée et précise. À l'occasion, il convient d'ajouter que la présence de ces arrière-fonds cosmologiques n'est pas une particularité des French theories du XX siècle : le XIX ${ }^{\mathrm{e}}$ siècle - positiviste, matérialiste, darwinien, anticlérical, marxiste - fut partout en Europe un âge d'or de l'aspiration sotériologique. Bien évidemment, l'infrastructure cosmologique à visée sotériologique n'est pas universelle dans le domaine des sciences sociales. Toutefois, elle y est récurrente, et il est possible de démontrer qu'elle sous-tend bon nombre des théories auxquelles on accorde volontiers le statut de "grandes"; ces sont aussi ces théories à vocation sotériologique qui suscitent, plus que les autres, des engouements enthousiastes à la fois dans le monde académiques et auprès du grand public.

Il serait probablement tentant - peut-être même rassurant - de penser que ces projets sotériologiques, ainsi que les visions du monde qui leur servent de socle, ne sont que des ornements rhétoriques ajoutés ex post à des savoirs construits exclusivement à partir de données empiriques et de considérations théoriques. Je crois que nous devrions envisager sérieusement une hypothèse inverse, selon laquelle les cosmologies à ambition sotériologique précèdent souvent le travail de recherche et peuvent exercer une influence déterminante - aussi bien négative que positive - sur les grands choix théoriques, les options épistémologiques, les sympathies et antipathies pour des modes de pensée, les types de sources utilisées, les postures rhétoriques, les procédures de validation, etc. C'est une hypothèse que je pourrais aisément corroborer à partir de mes quatre études de cas.

Quoi qu'il en soit, ces études de cas montrent que mes quatre éminents auteurs de "grandes théories " étaient engagés dans une double quête, vouée simultanément à la connaissance objective et à la transformation rédemptrice du monde humain. Cette double quête suscite trois ordres de questions : questions anthro-

Paris, L'Édition de l'Aube, 2003. 
pologiques, questions historiques et questions épistémologiques, très différentes les unes des autres, mais qui ont toutes leur importance.

Sur le plan anthropologique, il est intéressant de souligner certaines constantes qui se dessinent derrières une diversité des savoirs des sciences sociales. Les " grandes théories " manifestent majoritairement un intérêt prononcé pour la question du mal : elles s'interrogent sur les imperfections de la chose humaine et essaient de déterminer si ces imperfections sont ontologiques, inévitables, inscrites dans la nature des choses, ou si elles ne sont que des accidents de l'histoire, suscitées par la contingence culturelle et persistant à titre de conventions auxquelles il est éventuellement possible de mettre un terme. Les prolifiques débats sur la construction sociale de ceci ou de cela, qui durent depuis une trentaine d'années, ont pour enjeu principal l'extension du domaine de l'accidentel, du construit, au détriment de ce qui apparaissait jusqu'alors comme donnée, naturel, ontologiquement fixé. Si l'on doit reconnaître un mérite majeur au livre d'Ian Hacking Social Construction of What? ${ }^{22}$, c'est d'avoir montré que les discussions en apparence scolastiques sur la construction sociale, la déconstruction, le contingentisme, l'inevitabilisme, cherchent en fait à dessiner une nouvelle frontière entre ce qui, dans le monde où nous vivons, demeure intangible et ce qui a été fabriqué par les hommes et qui, de ce fait, pourrait être transformé, reformé, par les hommes : la race, l'ethnie, la nation, le genre, la nature, etc. Ces discussions sont donc à la fois ontologiques et politiques, car la frontière ontologique entre l'essentiel et l'accidentel fixe les limites infranchissables à l'action politique dont on peut raisonnablement espérer la réussite. Par exemple, l'échec du marxisme appliqué dans les pays de l'empire soviétique tenait, en grande partie, à une représentation erronée de la malléabilité de l'économie, de la vie sociale et de la personne humaine : son projet révolutionnaire d'une refondation du monde était tributaire - les travaux récents d'historiens le

22 Ian Hacking, Social Construction of What? Cambridge, Mass., Harvard University Press, 1999. 
montrent clairement - d'une vision du monde social fondée sur une ontologie singulière, où le domaine des propriétés essentielles, donc inaltérables, se trouvait réduit à une portion congrue.

Passons à présent à la dimension historique du phénomène en question. Sa prise en compte est nécessaire pour mieux comprendre l'ambition sotériologique qui accompagne les sciences sociales depuis leur fondation jusqu'à nos jours. Mon hypothèse - que j'ai commencée à étayer dans Anthropologies rédemptrices ${ }^{23}$ - est que le schème cosmologique sous-jacent aux théories des sciences sociales, ainsi que la finalité sotériologique à laquelle ces théories se trouvent souvent assujetties, s'inscrivent dans un réseau de filiations historiquement attestées qui unissent les sciences sociales aux idéologies politiques, systèmes philosophiques et doctrines théologiques antérieurs. Si récurrence historique il y a ici, elle dépasse largement les limites des sciences sociales ou des sciences tout court, embrassant une longue tradition millénariste, si typique de la culture occidentale, depuis l'émergence, au XVIII ${ }^{\mathrm{e}}$ siècle, de ce que l'historien Peter Gay a appelé, sans doute excessivement, le "paganisme moderne ", jusqu'aux " religions politiques " du XXe siècle, dotées de puissants relais philosophiques $^{24}$. Des historiens des idées, comme Isaiah Berlin et Leszek Kolakowski, ont naguère consacré à cette question des analyses très pénétrantes, qui mériteraient d'être reprises aujourd'hui à nouveaux frais. Une chose est sûre : si les sciences sociales restent si marquées par une orientation sotériologique, c'est parce qu'elles sont nées, au XIX ${ }^{\mathrm{e}}$ siècle, dans nos sociétés de tradition millénariste, qui ont cherché, à cette époque, à substituer à la vision du monde chrétienne non seulement des visions du monde laiques, mais aussi des sotériologies laïques : leurs nouvelles cosmologies sotériologiques reprenaient, de même que beaucoup de doctrines politiques de cette époque, des catégories de pensée théologique, laïcisée au XVIII ${ }^{\mathrm{e}}$ siècle par les philosophes des Lumières.

23 Wiktor Stoczkowski, Anthropologies rédemptrices, op. cit.

24 Peter Gay, The Enlightenment. The Rise of Modern Paganism, New York, W.W.

Norton \& Company, 1966. 
La troisième dimension du problème se situe sur le plan épistémologique. Et la première question qu'il est nécessaire de poser à cet égard est la suivante : quel est le prix, en termes de solidité et de validité empirique de leurs savoirs, que les sciences sociales payent pour s'être livrées, simultanément, à bâtir une connaissance empirique de la réalité humaine et à transformer profondément cette réalité? C'est la question des plus délicates. Mes études de cas suggèrent qu'il existe une incompatibilité partielle entre les deux objectifs de la double quête des sciences sociales. La production du savoir empiriquement fondé s'articule parfois mal avec l'ambition d'offrir des visions du monde marquées par un radicalisme sotériologique. Sur des exemples précis, il est possible de montrer que l'ambition cosmologique peut limiter fortement notre capacité à connaître et à comprendre le monde humain. Il faut pourtant se bien garder d'en faire des généralisations hâtives. Il est également possible - des exemples précis existent également - que les visions cosmologiques soient parfois porteuses d'idées nouvelles et pertinentes, qui peuvent engendrer des programmes de recherches fertiles. En même temps, des espérances sotériologiques peuvent fournir au chercheur, sur le plan psychologique, une motivation puissante, sans laquelle il est difficile, voire impossible, de livrer des efforts à proprement parler surhumains nécessaires pour bâtir de grandes théories véhiculées souvent par des œuvres qui sont grandes non seulement du point de vue qualitatif, mais surtout du point de vue quantitatif. À cela s'ajoute le problème de la demande sociale : le public intéressé par nos sciences continue à attendre d'elles des visions du monde et des visions laïques d'un salut immanent. Ainsi, en dépit des limites que ces visions peuvent imposer à nos capacités cognitives, il serait simpliste d'affirmer qu'elles ne sont qu'un obstacle à la connaissance, à l'instar des idola forii de Francis Bacon. La question est complexe et elle appelle des réponses nuancées, empiriquement étayées.

Une chose est certaine : les "grandes théories " interdisciplinaires qui débouchent sur des visions cosmologiques à tonalité sotériologique continuent à jouir d'une grande reconnaissance à 
la fois académique et extra-académique. Cet état des choses sera-t-il permanent?

À cet égard, au moins trois scenarii du développement possible de nos disciplines sont envisageables. Le premier est celui du maintien du status quo : les sciences sociales conserveront leur démarche traditionnelle, soumises au rythme des changements de paradigme comme la haute couture l'est aux changements de la mode; fournissant des visions du monde adaptées au goût du jour, elles continueront à être prisées à cause de leur capacité virtuose à construire des " discours " et " récits " où abondent des promesses que nul ne leur tiendra rigueur de ne pas satisfaire, puisqu'elles seront aussitôt chassées par d'autres, tout aussi bienpensantes et enivrantes que les précédentes. Dans cette hypothèse, il est possible qu'une partie des sciences sociales évolue vers une nouvelle forme de sophistique, fortement imprégnée des savoirfaire rhétoriques, comme le laisse présager, par exemple, le succès international de la socio-anthropologie politique de Bruno Latour, aujourd'hui l'auteur français vivant le plus cité dans le monde.

Le deuxième scénario part de l'hypothèse que les sciences sociales perdent leur quasi-monopole, acquis depuis le milieu du $\mathrm{XIX}^{\mathrm{e}}$ siècle, de la production des visions cosmologiques reconnues comme légitimes. En effet, elles sont désormais concurrencées dans ce domaine par des conceptions provenant de la culture non académique, voire de la culture populaire (il y a une dizaine d'années, j'ai consacré un livre aux visions du monde produites, dans la seconde moitié du XXe siècle, au sein d'une subculture très particulière, à laquelle je donne le nom d'occultiste), et dont l'impact sur nos sociétés, mesuré en termes quantitatifs, est désormais plus grand que l'influence des théories des sciences sociales : le volet le plus connu de cette subculture est qualifié souvent de mouvances New Age $e^{25}$. Dans cette deuxième hypothèse, l'émergence de nouveaux producteurs alternatifs des

$25 \quad$ Wiktor Stoczkowski, Des Hommes, des dieux et des extraterrestres. Ethnologie d'une croyance moderne, Paris, Flammarion, 1999. 
visions du monde ${ }^{26}$, conjuguée avec les pressions de plus en plus fortes exercées par les bailleurs de fonds, étatiques et privés, désireux de ne financer que la production des savoirs opératoires et vérifiables, peut conduire les sciences sociales à abandonner progressivement leurs ambitions cosmologiques et à se soumettre entièrement aux contraintes d'un modeste travail empirique, celui-ci devenant ainsi leur principal atout. Cela ne manquerait pas de laisser un territoire vide qu'une nouvelle classe d'idéologues pourrait investir, de même que les chercheurs en sciences sociales ont naguère investi le champ laissé libre par un affaiblissement des doctrines religieuses et par l'incapacité des théories philosophiques à s'y substituer. Dans ce cas-là, le domaine des sciences sociales pourrait voir des expertises remplacer progressivement des prophéties et visions du monde, proposées jusqu'alors sous l'étiquette de théories. Ceci est un scénario peut-être peu exaltant, mais sans nul doute optimiste.

Le troisième scénario, pessimiste mais plausible, part de la possibilité que les sciences sociales non seulement perdent leur monopole cosmologique, mais qu'elles s'avèrent incapables d'offrir aux sociétés qui les financent un savoir fondé et utile que celles-ci sont en droit d'attendre des chercheurs. Dans ce cas-là, il est envisageable que les sciences sociales disparaissent à une échéance plus ou moins courte, rejoignant dans les annales de l'histoire la longue liste des inventions éphémères que l'humanité a mises à l'essai pendant un bref laps du temps - deux siècles environ en ce qui concerne les sciences sociales - pour s'en passer fort bien par la suite.

Cette dernière possibilité est, malheureusement, tout à fait plausible, comme l'attestent les débats récents, dans plusieurs pays, sur le financement des sciences sociales en particuliers, ou des humanités en général. Cela incite particulièrement à établir un bilan lucide des acquis et des échecs des sciences sociales, depuis leur émergence jusqu'à aujourd'hui. Et quand on jette un

$\overline{26}$ Il en existe d'autres que les occultistes et les New Agers; les écologistes par exemple, les uns et les autres secondés formidablement, dans la diffusion de leurs idées, par les nouvelles technologies de la communication. 
regard en arrière, quel étrange spectacle nous apparaît! Que de promesses, que de séductions, que de rhétorique, que d'exaltantes fresques brossées à grands traits! La surenchère ne connaît aucune limite. C'est à qui annoncera la plus grande rupture, la plus profonde refondation, le plus brillant changement de paradigme, l'innovation la plus neuve, la purification la plus radicale du mal. Pourtant, il n'est pas certain que le chemin de la connaissance soit nécessairement pavé de bonnes intentions et que l'espérance reste toujours la meilleure compagne de la compréhension. Il est sans doute exaltant de prodiguer des promesses millénaristes et d'imaginer que l'on s'attaque à la racine même du mal; il est même fort probable que le public escompte du chercheur qu'il adopte une telle posture messianique. Cependant, le chercheur ne devrait pas oublier que sa mission première est de décrire et de comprendre le « monde tel qu'il est ", au lieu de le critiquer et d'imaginer des arrières mondes possibles, en s'obstinant à voir les problèmes du présent comme manifestations d'un mal radical, dont l'existence appelle un renouvellement rédempteur.

\section{Bibliographie}

Bourdieu, Pierre, "Genèse et structure du champ religieux ", Revue française de sociologie, vol. 12, n 3, 1971, p. 295-334.

Bourdieu, Pierre, "Le champ scientifique ", Actes de la recherche en sciences sociales, vol. 2, $\mathrm{n}^{\text {os }} 2-3,1976$, p. 88-104.

Bourdieu, Pierre, Leçon sur la leçon, Paris, Minuit, 1982.

Bourdieu, Pierre, Ce que parler veut dire, Paris, Fayard, 1982.

Bourdieu, Pierre, Raisons pratiques, Paris, Seuil, 1994.

Bourdieu, Pierre et Yvette Delsaut, « Le couturier et sa griffe : contribution à une théorie de la magie ", Actes de la recherche en sciences sociales, vol. $1, \mathrm{n}^{\circ} 1,1975$, p. 7-36.

Bubloz, Yvan, «Augustin et Porphyre sur le salut : pour une comparaison analogique et non apologétique du christianisme et du néoplato- 
nisme ", dans Maya Burger et Claude Calame (dir.), Comparer les comparatismes. Perspectives sur l'histoire et les sciences des religions, Paris, Edidit, 2006, p. 113-140.

Durkheim, Émile, Le Suicide. Étude de sociologie, Paris, Alcan, 1897.

Durkheim, Émile, Les Formes élémentaires de la vie religieuse, Paris, Alcan, 1912.

Gay, Peter, The Enlightenment. The Rise of Modern Paganism, New York, W.W. Norton \& Company, 1966.

Hacking, Ian, Social Construction of What? Cambridge, Mass., Harvard University Press, 1999.

Latour, Bruno, Changer de société - refaire de la sociologie, Paris, La Découverte, 2006.

Latour, Bruno, Un Monde pluriel mais commun, entretiens avec François Ewald, Paris, L'Édition de l'Aube, 2003.

Lévi-Strauss, Claude, « Race et culture ", dans Le Regard éloigné, Paris, Plon, [1971] 1983, p. 377-222.

Lévi-Strauss, Claude, "Discours de récipiendaire à la remise du prix Erasme ", dans Praemium Erasmianum MCMLXXIII, Amsterdam, Stichting Praemium Erasmianum, 1973, p. 24-28.

Stoczkowski, Wiktor, Des Hommes, des dieux et des extraterrestres. Ethnologie d'une croyance moderne, Paris, Flammarion, 1999.

Stoczkowski, Wiktor, Anthropologies rédemptrices. Le monde selon LéviStrauss, Paris, Hermann, 2008. 